\title{
Folliculotropic mycosis fungoides: challenging clinical, histopathological and immunohistochemical diagnosis*
}

\author{
Maria Isabel Braga Malveira ${ }^{1}$ \\ Shirley Braga Lima Gamonal ${ }^{1,2}$
}

\author{
Gianne Pascoal ${ }^{1}$ \\ Maria Christina Marques Nogueira Castañon ${ }^{3}$
}

DOI: http://dx.doi.org/10.1590/abd1806-4841.20175634

\begin{abstract}
Mycosis fungoides is a cutaneous T-cell lymphoma with various clinical and pathological presentations. Early lesions are nonspecific, which hinders early diagnosis. The folliculotropic subtype is manifested as acneiform lesions, follicular papules or erythematous plaques mainly on the face, neck and upper trunk. Histopathology shows dense lymphocytic infiltrate surrounding and infiltrating the hair follicles. A case of difficult histopathological diagnosis with florid and unusual skin lesions mainly on the face is reported.
\end{abstract}

Keywords: Diagnosis; Lymphoma, T-Cell, cutaneous; Mycosis fungoides

\section{INTRODUCTION}

Mycosis fungoides is considered the most common type of cutaneous T-cell lymphoma, despite being rare. ${ }^{1}$ Early diagnosis of the condition is challenging for dermatologists because the initial clinical and histopathological features can be unspecific. It has a prolonged course, sometimes mimicking benign dermatoses. ${ }^{2}$ Folliculotropic mycosis fungoides is the most common variant. It can present with plaques, acneiform lesions or as a tumor, being alopecia and eyebrow involvement typical. On histology, there is a dense lymphocytic infiltrate around hair follicles, sometimes with destruction of the follicle. In the early phases, there is a mild perivascular inflammatory infiltrate in the upper dermis with no obvious lymphocyte atypia. ${ }^{3}$ The prognosis of this type of mycosis fungoides is poorer, with the survival ranging from 70 to $80 \%$ in five years. ${ }^{4}$ The authors describe a case with unusual and florid clinical presentation, of difficult histopathological and immunohistochemical diagnosis.

\section{CASE REPORT}

A 63-year-old dark-skinned male patient, hypertensive, reported the appearance of erythematous-brown, edematous and itchy plaques on the abdomen in the beginning of 2013, with inconclusive histopathology and immunohistochemistry. He remained under follow-up and presented to the outpatient dermatology clinic in February 2015 with an ulcerated tumor on the skin of the nose and worsening of the previous lesions. On clinical examination, he had brown and edematous plaques on the frontal region, associated with alopecia of the eyebrows and hairline, in addition to a deforming tumor on the nose (Figure 1). ENT assessment demonstrated an infiltrative lesion, with total obstruction of the right nostril and partial of the left nostril, with associated mucoid discharge. No lesions on the palate or jugal mucosa were seen. Slightly lichenified brown plaques were seen on the upper and lower limbs, predominantly on the trunk and ulcerated tumors on the upper limbs (Figure 2).

Study submitted in 28.01.2016

Approved by the Advisory Board and accepted for publication on 12.06.2016

* Study conducted at Núcleo de Pesquisa em Dermatologia, Hospital Universitário da Universidade Federal de Juiz de Fora (NUPEDE -HU-UFJF) - Juiz de Fora (MG), Brazil.

Financial Support: None.

Conflict of Interests: None.

Department of Dermatology, Hospital Universitário da Universidade Federal de Juiz de Fora (HU-UFJF) - Juiz de Fora (MG), Brazil.

Outpatient Clinic of Dermatology, Hospital Regional João Penido - Fundação Hospitalar do Estado de Minas Gerais (HRJP-FHEMIG) - Juiz de Fora (MG), Brazil.

Department of Morphology, Universidade Federal de Juiz de Fora (UFJF) - Juiz de Fora (MG), Brazil.

(C)2017 by Anais Brasileiros de Dermatologia 
There was no lymphadenopathy. The suspicion was of paracoccidioidomycosis, leishmaniasis, syphilis, leprosy, sarcoidosis, mycosis fungoides, and lethal midline granuloma. Blood samples were collected, and microscopy, Montenegro's test, tuberculin test, bacilloscopy, serology for hepatitis, HIV and syphilis were all performed, and all were negative. Chest radiograph and abdomen ultrasound were unremarkable. New biopsies were taken, with unspecific findings. The patient's follow-up was then irregular, with long intervals between appointments, with worsening of the tumor on the nose. In July 2015 three new biopsies from different skin lesions were taken and the histopathology of the abdominal plaque revealed a dense atypical mononuclear infiltrate, involving the remaining follicular sheath and eccrine glands associated to psoriasiform hyperplasia of the epidermis and granulomatous reaction (Figure 3). Immunohistochemistry favored the diagnostic interpretation of non-Hodgkin epidermotropic and folliculotropic T-cell cutaneous lymphoma, CD4 and CD30 positive (Figures 4 and 5). The patient was seen by the hematology team and chemotherapy with gemcitabine and interferon, associated to phototherapy were scheduled.

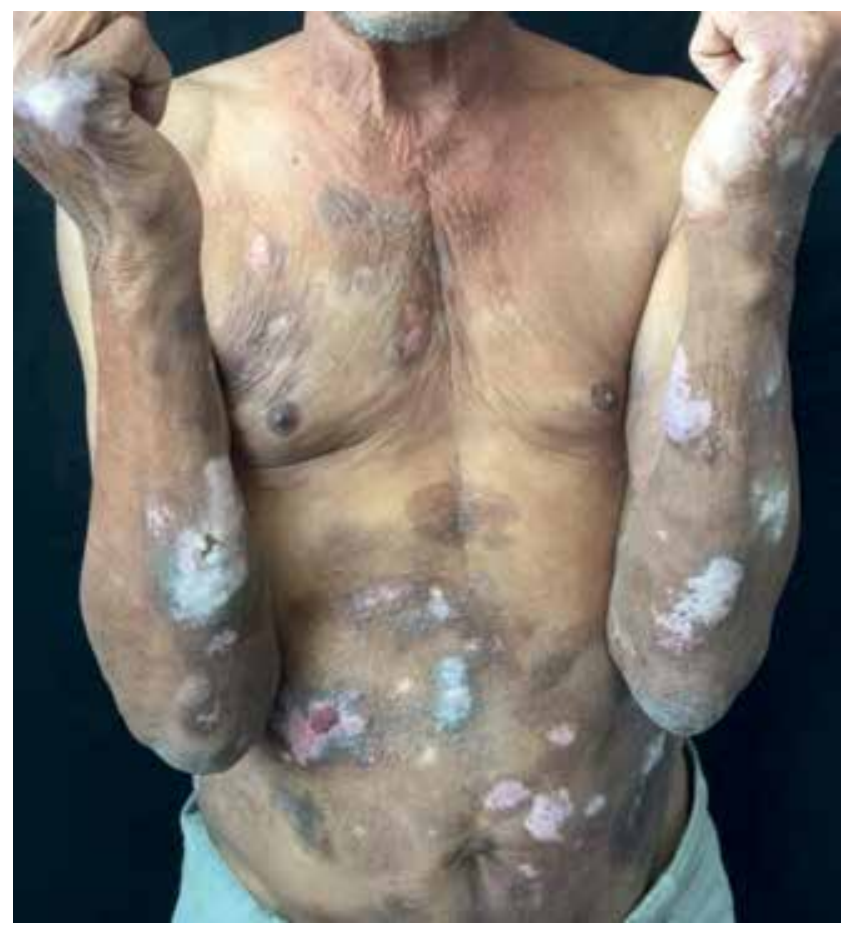

Figure 2: Plaques on the trunk and tumors on the upper limbs

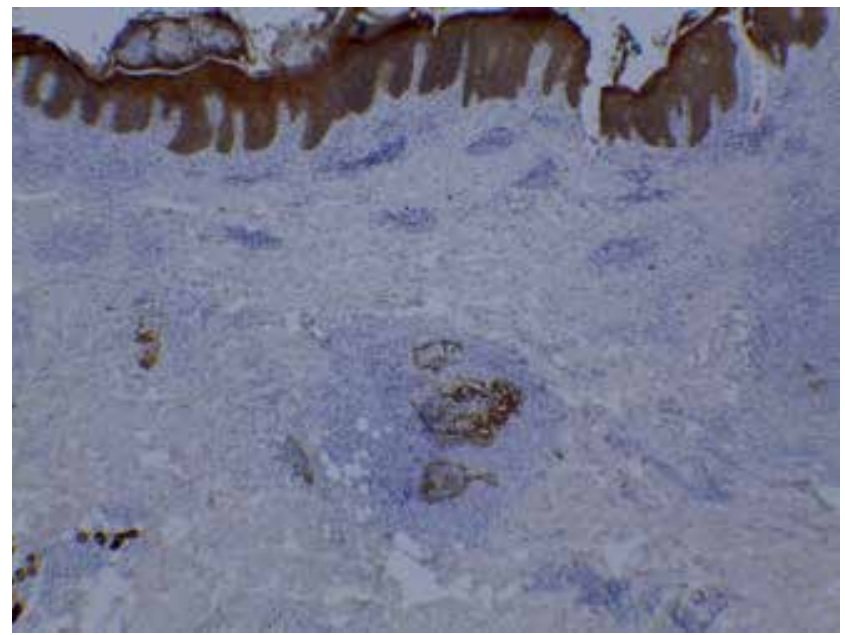

FIGURE 4: Cytokeratin (AE1/3) positivity in epithelial elements, highlighting the destruction of the adnexal epithelium by tumor cells (Immunohistochemistry, X40)
Figure 3: Psoriasiform epidermal hyperplasia, predominantly lymphocytic folliculotropic infiltrate, loose epithelioid granulomas (A: Hematoxylin \& eosin, X100); lymphocytic infiltrate involving the remaining external follicular sheaths (B: Hematoxylin \& eosin, X100)

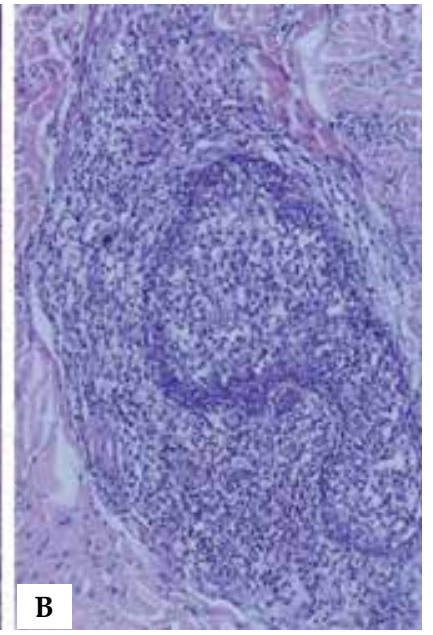

An Bras Dermatol. 2017;92(5 Suppl 1):73-5. 

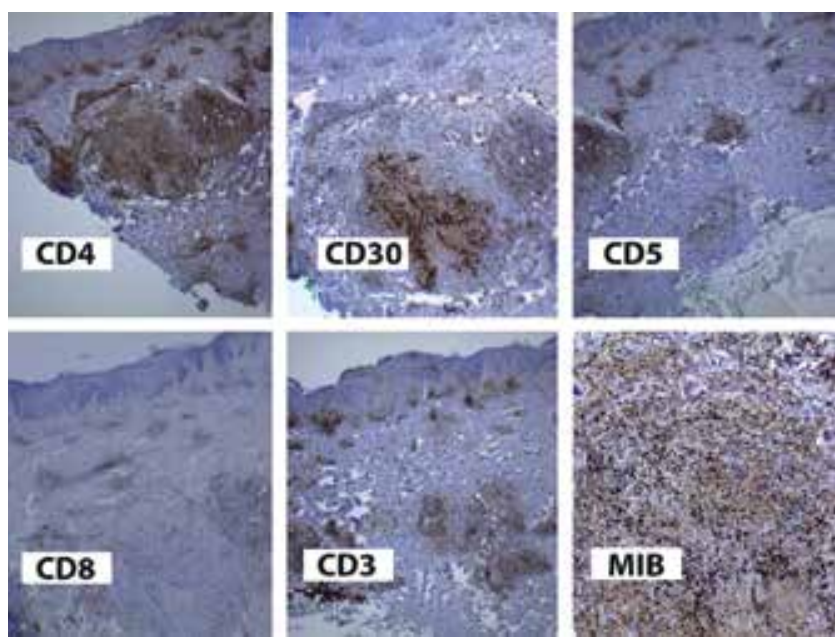

FIGURE5: Predominance of CD4+ population(Immunohistochemistry, X40), mainly of large cells; CD30+ (Immunohistochemistry x100) of large cells with more atypia around vessels and appendages; CD8+ (Immunohistochemistry, X40) in some lymphocytes; CD3+ e CD5+ (Immunohistochemistry, X100) in most lymphoid cells; Ki-67+ (MIB1) in about $50 \%$ of nuclei (Immunohistochemistry, X400)

\section{DISCUSSION}

Mycosis fungoides is a subtype of T-cell cutaneous lymphoma, with an incidence of 0.5 case per 100,000 persons per year. It has multiple clinical and pathological variants and affects adults between 55 and 60 years of age, with slight male predominance. ${ }^{5}$

The most recent classification of the World Health Organization and of the European Organization of Research and Treatment of Cancer describes the classical form of Alibert-Bazin and three variants: folliculotropic, pagetoid reticulosis and granulomatous slack skin. ${ }^{6}$ In folliculotropic mycosis fungoides, the initial lesions have

\section{REFERENCES}

1. Kim YH, Hoppe RT. Mycosis fungoides and the Sezary syndrome. Semin Oncol. 1999:26:276-89.

2. Bergman R, Faclieru D, Sahar D, Sander CA, Kerner H, Ben-Aryeh $\mathrm{Y}$, et al. Immunophenotyping and T-cell receptor gamma gene rearrangement analysis as an adjunct to the histopathologic diagnosis of mycosis fungoides. J Am Acad Dermatol. 1998;39:554-9.

3. Flaig MJ, Cerroni L, Schuhmann K, Bertsch HP, Kind P, Kaudewitz P, et al. Follicular mycosis fungoides: a histopathologic analysis of nine cases. J Cutan Pathol. 2001;28:525-30.

4. van Doorn R, Scheffer E, Willemze R. Follicular mycosis fungoides: a distinct disease entity with or without associated follicular mucinosis. Arch Dermatol. 2002;138:191-8.

5. Willemze R, Jaffe ES, Burg G, Cerroni L, Berti E, Swerdlow SH, et al. WHO-EORTC classification for cutaneous lymphomas. Blood. 2005;105:3768-85.

6. Swerdlow SH, Campo E, Harris NL, Jaffe ES, Pileri SA, Stein H, et al. WHO Classification of Tumours of Haematopoietic and Lymphoid Tissues. Lyon, France: IARC Press; 2008.

7. Gerami P, Rosen S, Kuzel T, Boone SL, Guitart J. Folliculotropic mycosis fungoides. An aggressive variant of cutaneous T-cell lymphoma. Arch Dermatol. 2008;144:738-46. an acneiform aspect, or show follicular papules and erythematous plaques preferentially on the face, neck, and upper trunk. The heavy involvement of the hair follicle with follicular hyperplasia can result in the formation of tumor-like lesions even in the absence of a true tumor. Eyebrow involvement with alopecia is typical. With disease progression, there is the formation of bulky tumor masses and the face acquires a leonine appearance. ${ }^{7}$

On histopathology, the folliculotropic variant shows a dense inflammatory infiltrate that surrounds and infiltrates the hair follicle and usually spares the interfollicular epidermis. There is epidermotropism with folliculotropism in approximately $10 \%$ of the cases. Typically, the follicles show a corneal plug and sometimes mucin degeneration of the epithelium with variable intensity. There can be infiltration of the eccrine sweat glands. The immunophenotype is similar to classic mycosis fungoides, with atypical CD4+ and CD45RO+ T-cells, with frequent loss of the T-cell surface antigen, such as CD2, CD5 and/or CD7. ${ }^{8}$ Large CD30+ blast cells can be seen interspersed within the infiltrate. ${ }^{4}$ The histologic pattern of the reported patient demonstrated granulomatous reaction associated to the folliculotropic pattern with $\mathrm{CD} 30+$ cells, which are uncommon findings. An important differential diagnosis is the extranodal NK/ T-cell lymphoma nasal-type, that predominantly affects the nasal cavity and nasopharynx but whose cells are CD4 and CD5 negative. ${ }^{4}$

For the treatment of early disease, topical medications are used, such as corticosteroids, bexarotene and nitrogen mustard, as well as phototherapy or electron beam therapy. Other modifiers of the biological response, such as interferon alpha, can be used for advanced disease. Polychemotherapy regimens are indicated in cases with lymph node and/or visceral involvement, and for the cases refractory to topical treatment. ${ }^{9,10}$ Due to the deeper location of the infiltrate, folliculotropic mycosis fungoides is less responsive to treatments directed to the skin. ${ }^{4} \square$
8. Bernengo MG, Novelli M, Quaglino P, Lisa F, De Matteis A, Savoia P, et al. The relevance of the $\mathrm{CD} 4+\mathrm{CD} 26$ - subset in the identification of circulating Sézary cells. Br J Dermatol. 2001;144:125-35.

9. Whittaker SJ, Marsden JR, Spittle M, Russell Jones R; British Association of Dermatologists; U.K. Cutaneous Lymphoma Group. Joint British Association of dermatologists and UK Cutaneous Lymphoma Group guidelines for the management of primary cutaneous T-cell lymphomas. $\mathrm{Br} \mathrm{J}$ Dermatol. 2003;149:1095-1107.

10. Dummer R, Kempf W, Hess Schmid M, Häffner A, Burg G. Therapy of cutaneous lymphoma: current practice and future developments. Onkologie. 2003;26:366-72.

\author{
MAILING ADDRESS: \\ Maria Isabel Braga Malveira \\ R. Eugênio do Nascimento, s/n \\ Dom Bosco \\ 36038-330 Juiz de Fora, MG \\ E-mail: belmalveira@hotmail.com
}

How to cite this article: Malveira MIB, Pascoal G, Gamonal SBL, Castañon MCMN. Folliculotropic mycosis fungoides: challenging clinical, histopathological and immunohistochemical diagnosis. An Bras Dermatol. 2017;92(5 Suppl 1): 73-5. 